\title{
HOMOTOPY IN FUNCTOR CATEGORIES
}

\author{
BY
}

ALEX HELLER

\begin{abstract}
If $\mathbf{C}$ is a small category enriched over topological spaces the category $\boldsymbol{T} \mathbf{C}$ of continuous functors from $\mathbf{C}$ into topological spaces admits a family of homotopy theories associated with closed subcategories of $\mathbf{C}$. The categories $\mathcal{T} \mathbf{C}$, for various $\mathbf{C}$, are connected to one another by a functor calculus analogous to the $\otimes$, Hom calculus for modules over rings. The functor calculus and the several homotopy theories may be articulated in such a way as to define an analogous functor calculus on the homotopy categories. Among the functors so described are homotopy limits and colimits and, more generally, homotopy Kan extensions. A by-product of the method is a generalization to functor categories of E. H. Brown's representability theorem.
\end{abstract}

Introduction. The subject of this investigation is the kind of homotopy theory that can be done in categories $\mathcal{T}^{\mathbf{C}}$ of functors with values in the category of topological (more properly, separated compactly generated) spaces. The index category $\mathbf{C}$ is itself supposed to be enriched over $\mathcal{T}$ so that such categories as, for example, that of $G$-spaces where $G$ is a topological monoid or group, are subsumed.

There are two points which are to be taken into account. First, in such a functor category there are always several notions of homotopy. Two of these have been extensively considered [3, 13, 14]; a third, provided by the obvious homotopy congruence, has perhaps been thought too trivial to warrant much interest. In fact there are many more-one, indeed, for each closed subcategory of the index category. Second, these functor categories are related to one another by a functor calculus formally analogous to the "tensor product" and "hom" calculus of module theory. In this calculus appear, in particular, the functors induced by composition as well as their adjoints, the Kan extensions.

The objective then is to articulate these notions of homotopy and the functor calculus into a comprehensive system. As usual this is mediated by the introduction of notions of fibration and cofibration. The several "standard" forms in which this may be done-most notably Quillen's "model category" structures [10, cf. also 4]-are perhaps not adequate here. It has, rather, seemed appropriate to associate to each notion of homotopy two each of fibration and cofibration: a kind of bifurcation of function not unfamiliar in such instances as this of generalization.

There are to be then weaker and stronger notions of fibration and cofibration. It is the stronger ones which will interact well with the functor calculus. To show that

Received by the editors February 11, 1981.

1980 Mathematics Subject Classification. Primary 55U35; Secondary 18A25, 18G55.

Key words and phrases. Abstract homotopy, functor category, homotopy limit, homotopy Kan extension, half-exact functor. 
there are enough of them is the task of a central "mapping cylinder" theorem which will be perceived as originating in Steenrod's version of the Milgram bar construction [12]. Using this, it is possible to test the comprehensive system by proving the existence of various adjoint functors on homotopy categories, such as homotopy limits and homotopy Kan extensions [1, 3, 13, 14]. A test of a different sort is provided by a generalization to functor categories of E. H. Brown's representability theorem for half-exact functors.

Recent work $[2,6,9]$ has exhibited homotopy theories as arising in a variety of contexts not lending themselves to the standard treatments. The present discussion may perhaps serve to reinforce the notion that it may yet be premature to decide just what it is that constitutes the formal structure of such a theory.

1. The functor calculus for $\mathcal{T}$-categories. Let us denote by $\mathcal{T}$ the category of separated compactly generated spaces. $\mathcal{T}$ is complete, cocomplete and cartesianclosed; we write $(-)^{X}$ for the function-space functor right adjoint to $-\times X$. Moreover, $\mathcal{T}$ contains all $\mathrm{CW}$ complexes.

By a $\mathcal{T}$-category we mean an internal category of $\mathcal{T}$ with a discrete object-space or, equivalently, a small category enriched over $\mathcal{T}$, that is to say provided with a T-topology on each hom-set such that composition is continuous. If $\mathbf{C}, \mathbf{D}$ are T-categories we mean by a functor $F: \mathbf{C} \rightarrow \mathbf{D}$ a functor on the underlying categories such that for each $c, d \in \mathbf{C}_{0}=$ ob $\mathbf{C}$, the map $\mathbf{C}(c, d) \rightarrow \mathbf{D}\left(F_{c}, F_{d}\right)$ is continuous.

Any small category $\mathbf{C}$ may be regarded as a $\mathcal{T}$-category by giving to each $\mathbf{C}(c, d)$ the discrete topology. We call such categories $\mathcal{T}$-discrete, in contrast to discrete categories, for which $\mathbf{C}(c, d)=\varnothing, c \neq d$ and $\mathbf{C}(c, c)=\left\{1_{c}\right\}$.

If $\mathbf{C}$ is a $\mathcal{T}$-category $\mathcal{T}^{\mathbf{C}}$ denotes the category of functors $X: \mathbf{C} \rightarrow \mathcal{T}$ which are continuous in the sense that for each $c, d \in \mathbf{C}_{0}$ the map $\mathbf{C}(c, d) \rightarrow X_{d}^{X_{c}}$ is continuous, or equivalently its transpose $\mathbf{C}(c, d) \times X_{c} \rightarrow X_{d}$ is continuous.

Ends and coends of functors into $\mathcal{T}$ are defined in the usual way (cf. Mac Lane [8]); the following statement is easily verified.

Proposition 1.1. If $X: \mathbf{C}^{\mathrm{op}} \times \mathbf{C} \times \mathbf{D} \rightarrow \mathcal{T}$ is continuous then the functors $\mathbf{D} \rightarrow \mathcal{T}$ defined by

$$
d \mapsto \int_{c} X_{c, c, d}, \quad d \mapsto \int^{c} X_{c, c, d}
$$

are also continuous.

Using these we may construct a calculus on the functor categories $\mathcal{T}^{\mathbf{c}}$, formally analogous to the calculus of modules over rings. The functorial pairing $\otimes_{\mathbf{C}}$ : $\mathcal{T}^{\mathbf{B} \times \mathbf{C}^{\text {op }}} \times \mathcal{T}^{\mathbf{C} \times \mathbf{D}} \rightarrow \mathcal{T}^{\mathbf{B} \times \mathbf{D}}$ is given by

$$
\left(X \otimes_{\mathrm{C}} Y\right)_{b, d}=\int^{c} X_{b, c} \times Y_{c, d} .
$$

Similarly, Hom $_{\mathbf{C}}:\left(\mathcal{T}^{\mathbf{B} \times \mathbf{C}}\right)^{\text {op }} \times \mathcal{T}^{\mathbf{C} \times \mathbf{D}} \rightarrow \mathcal{T}^{\mathbf{B}^{\text {op }}} \times \mathbf{D}$ is given by

$$
\operatorname{Hom}_{\mathbf{C}}(X, Y)_{b, d}=\int_{c} Y_{c, d}^{X_{b, c}} .
$$


These pairings are easily seen to satisfy the familiar-looking relations

$$
\begin{gathered}
\left(X \otimes_{\mathbf{C}} Y\right) \otimes_{\mathbf{D}} Z \approx X \otimes_{\mathbf{C}}\left(Y \otimes_{\mathbf{D}} Z\right), \\
\operatorname{Hom}_{\mathbf{D}}\left(X \otimes_{\mathbf{C}} Y, Z\right) \approx \operatorname{Hom}_{\mathbf{C}}\left(X, \operatorname{Hom}_{\mathbf{D}}(Y, Z)\right)
\end{gathered}
$$

for suitable $X, Y, Z$.

Hom $_{\mathbf{C}}$ gives $\mathcal{T}^{\mathbf{C}}$ an enrichment over $\mathcal{T}$. In particular, denoting by $*$ the constant

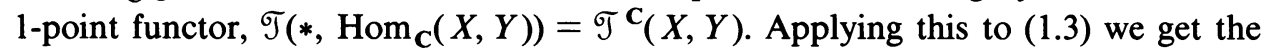
adjointness relation

$$
\mathcal{T}^{\mathbf{D}}\left(X \otimes_{\mathbf{C}} Y, Z\right) \approx \mathcal{T}^{\mathbf{C o p}}\left(X, \operatorname{Hom}_{\mathbf{D}}(Y, Z)\right)
$$

for $X: \mathbf{C}^{\text {op }} \rightarrow \mathcal{T}, Y: \mathbf{C} \times \mathbf{D} \rightarrow \mathcal{T}, Z: \mathbf{D} \rightarrow \mathcal{T}$.

The Yoneda lemma for continuous functors $\mathbf{C} \rightarrow \mathcal{T}$ is proved by the same argument as the standard one. If we denote by $\mathbf{C}: \mathbf{C}^{\mathrm{op}} \times \mathbf{C} \rightarrow \mathcal{T}$ the obvious continuous functor then, in our notation, it asserts that $\operatorname{Hom}_{\mathbf{C}}(\mathbf{C}, X) \approx X$. By the adjointness (1.3) this implies

$$
\mathbf{C} \otimes_{\mathbf{C}} X \approx X
$$

In particular $(X, Y) \rightarrow Y^{X}=\operatorname{Hom}_{\mathbf{C}}(\mathbf{C} \times X, Y)$ gives $\mathcal{T}^{\mathbf{C}}$ the structure of a cartesian closed category, since

$$
\mathcal{T}^{\mathbf{c}}\left(Z, \operatorname{Hom}_{\mathbf{C}}(\mathbf{C} \times X, Y)\right) \approx \mathcal{T}^{\mathbf{c}}\left(Z \otimes_{\mathbf{C}^{\mathrm{op}}}(\mathbf{C} \times X), Y\right) \approx \mathcal{T}^{\mathbf{c}}(Z \times X, Y) .
$$

We shall make a convention of denoting, for a space $W$ in $\mathcal{T}$, the constant functors with values $W$ by the same letter. From (1.2) we see directly that for suitable $X, Y$

$$
\begin{gathered}
(X \times W) \otimes_{\mathbf{C}} Y \approx\left(X \otimes_{\mathbf{C}} Y\right) \times W \approx X \otimes_{\mathbf{C}}(Y \times W), \\
\operatorname{Hom}_{\mathbf{C}}(X \times W, y) \approx \operatorname{Hom}_{\mathbf{C}}(X, Y)^{W} \approx \operatorname{Hom}_{\mathbf{C}}\left(X, Y^{W}\right) .
\end{gathered}
$$

If $F: \mathbf{C} \rightarrow \mathbf{D}$ then the functor $F^{*}: \mathcal{T}^{\mathbf{D}} \rightarrow \mathcal{T}^{\mathbf{C}}$ and its adjoints, the left and right Kan extensions along $F$, are given by the formulae

$$
\begin{gathered}
F^{*} Y=\mathbf{D} \circ(1 \times F) \otimes_{\mathbf{D}} Y \approx \operatorname{Hom}_{\mathbf{D}}(\mathbf{D} \circ(F \times 1), Y), \\
\operatorname{Lan}_{F} X=\mathbf{D} \circ(F \times 1) \otimes_{\mathbf{C}} X, \quad \operatorname{Ran}_{F} X=\operatorname{Hom}_{\mathbf{C}}(\mathbf{D} \circ(1 \times F), X) .
\end{gathered}
$$

In particular, if $W$ is constant as above then (1.5) implies

$$
\begin{aligned}
F^{*}(Y \times W) & \approx F^{*}(Y) \times W, & \operatorname{Lan}_{F}(X \times W) & \approx\left(\operatorname{Lan}_{F} X\right) \times W, \\
F^{*}\left(Y^{W}\right) & \approx\left(F^{*} Y\right)^{W}, & \operatorname{Ran}_{F}\left(Y^{W}\right) & \approx\left(\operatorname{Ran}_{F} Y\right)^{W}
\end{aligned}
$$

with the special case $\left(Y^{W}\right)_{c}=\left(Y_{c}\right)^{W}, c \in \mathbf{C}_{0}$.

2. Homotopy in functor categories. We denote by $I$ the unit interval $[0,1]$ and thus also, by our convention, a constant functor with that value. If $\mathbf{C}$ is a $\mathcal{T}$-category a homotopy in $\mathcal{T}^{\mathbf{C}}$ is a morphism $F: X \times I \rightarrow Y$ or equivalently its transpose $\tilde{F}$ : $X \rightarrow Y^{I}$; it is of course a homotopy of $F i_{0}=\mathrm{ev}_{0} \tilde{F}$ and $F i_{1}=\mathrm{ev}_{1} \tilde{F}$, where $i_{\alpha}, \mathrm{ev}_{\alpha}$ are the injections $X \rightarrow X \times I$ and the evaluations $Y^{I} \rightarrow Y$ at the endpoints of $I$. The existence of a homotopy defines the homotopy congruence $\sim$ in $\mathscr{T} \mathbf{C}$. The quotient category $\mathcal{T}^{\mathbf{C}} / \sim$ is the homotopy category Ho $\mathcal{T}^{\mathbf{C}}$. A morphism in $\mathcal{T}^{\mathbf{C}}$ is a homotopy 
equivalence if its image in $\mathrm{Ho}^{\mathrm{T}} \mathbf{C}$ is an isomorphism. A standard argument shows that $\sigma^{\mathbf{C}} \rightarrow \mathrm{Ho} \mathrm{\sigma}^{\mathbf{C}}$ is also the category of fractions with respect to the homotopy equivalences.

In $\mathcal{T}^{\mathbf{C}}$ we define cofibrations and fibrations by the usual homotopy extension and homotopy lifting properties. The mapping cylinder and cocylinder constructions for a morphism $f: X \rightarrow Y$, viz. the pushout and pullback

$\begin{array}{cccccc}X \sqcup X & \stackrel{\left(i_{0} i_{1}\right)}{\rightarrow} & X \times I & M^{f} & \rightarrow & Y^{I} \\ f \sqcup 1 \downarrow & & \downarrow & \downarrow & & \downarrow\left(\mathrm{ev}_{0} \mathrm{ev}_{1}\right) \\ Y \sqcup X & \rightarrow & M_{f} & Y \times X & \rightarrow & Y \times Y\end{array}$

give rise to factorizations of $f$ as a cofibration followed by a homotopy equivalence and as a homotopy equivalence followed by a fibration. Compositions of cofibrations are cofibrations. A pushout of a cofibration along any morphism is again a cofibration; we shall refer to the resulting square diagram as a $c$-pushout. Fibrations behave in dual fashion, giving rise to $f$-pullbacks.

The following assertions are proved by the arguments familiar in the case $\mathbf{C}=\mathbf{1}$, i.e., in $\mathcal{T}$.

Proposition 2.1. (i) A pushout of a cofibration which is also a homotopy equivalence is again a homotopy equivalence.

(ii) $A$ pushout of a homotopy equivalence along a cofibration is a homotopy equivalence.

(iii) If, in a morphism of c-pushouts, the three initial arrows are homotopy equivalences then so also is the fourth.

The dual statements for fibrations hold as well.

By a 2-cofibration we mean a commutative diagram

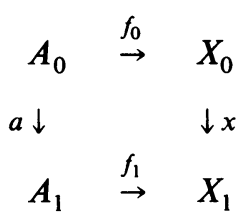

in which $a$ and $f_{0}$, as well as the canonical morphism $P \rightarrow X_{1}$, where $P$ is the pushout of $a$ and $f_{0}$, are all cofibrations. It follows of course that $x$ and $f_{1}$ are cofibrations too. 2-fibrations are defined dually.

It is sometimes convenient to think of cofibrations in $\mathcal{T}^{\mathbf{C}}$ as special objects in $\sigma^{\mathbf{C} \times \mathbf{2}}$, where $\mathbf{2}=(0 \rightarrow 1)$. Then $\mathbf{2}$-cofibrations are (in two ways) special morphisms between cofibrations, viz., $\left(f_{0}, f_{1}\right): a \rightarrow x$ and $(a, x): f_{0} \rightarrow f_{1}$ in (2.2).

Proposition 2.3. (i) Compositions of 2-cofibrations are 2-cofibrations.

(ii) A pushout in $\mathcal{T}^{\mathbf{C} \times \mathbf{2}}$ of a 2-cofibration in $\mathcal{T}^{\mathbf{C}}$ along a morphism of cofibrations is again a 2-cofibration. 
The dual statements for 2-fibrations also hold.

Coproducts of cofibrations are cofibrations, products of fibrations are fibrations and both products and coproducts of homotopy equivalences are homotopy equivalences. We shall see that these classes also have closure properties with respect to certain "infinite compositions".

Let us consider ordinal numbers $\beta$ as ordered sets and thus as categories-as for example 2 above. Thus $\beta_{0}$ is the underlying set of $\beta$, e.g. $\boldsymbol{2}_{0}=2$. A limit ordinal is then the colimit of its predecessors.

Proposition 2.4. If $\beta$ is a limit ordinal, $X: \beta \rightarrow \mathcal{T}^{\mathbf{C}}$ is colimit preserving and, for all $\alpha<\beta, X_{\alpha} \rightarrow X_{\alpha+1}$ is a cofibration then so also are $X_{\alpha} \rightarrow \operatorname{colim} X$ and, for all $\alpha<\gamma<\beta$, all $X_{\alpha} \rightarrow X_{\gamma}$.

We refer to this condition as closure under right infinite composition. The class of cofibrations which are also homotopy equivalences is also closed under right infinite composition. Dually, the classes of fibrations and of fibrations which are homotopy equivalences are closed under left infinite composition.

Proposition 2.5. The 2-cofibrations in $\mathcal{T}^{\mathbf{C}}$ are closed under right infinite composition in $\sigma^{\mathbf{C} \times 2}$. 2-fibrations are closed under left infinite composition.

Suppose that $X, Y: \beta \rightarrow \mathcal{T}^{\mathbf{C}}$ preserve colimits, $f: X \rightarrow \mathcal{T}$ and that, for all $\alpha<\beta$, $\left(f_{\alpha}, f_{\alpha+1}\right)$ is a 2 -cofibration, $\beta$ being a limit ordinal. Consider the diagrams

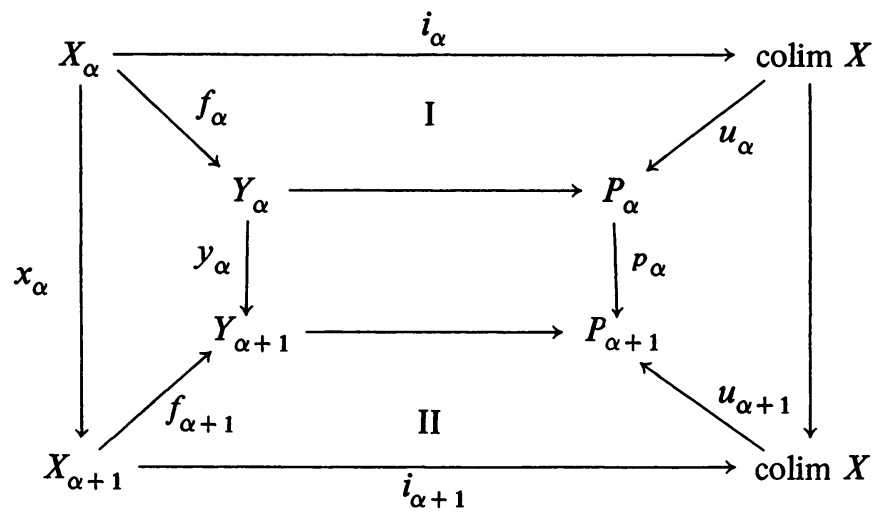

in which the squares I, II are pushouts. Then $\left(u_{\alpha}, u_{\alpha+1}\right)$ is the pushout, in $\sigma^{\mathbf{C} \times 2}$, of $\left(f_{\alpha}, f_{\alpha+1}\right)$ along $\left(i_{\alpha}, i_{\alpha+1}\right)$. Thus $\left(u_{\alpha}, u_{\alpha+1}\right)$ is by Proposition 2.3 a $\mathcal{T}$-cofibration, hence also $\left(1, p_{\alpha}\right)$; in particular $p_{\alpha}$ is a cofibration. By Proposition $2.4, P_{\alpha} \rightarrow \operatorname{colim} P$ is a cofibration. But colim ${ }_{\alpha<\beta} P_{\alpha}=$ colim $Y$, so that

is a 2-cofibration.

$$
\begin{array}{ccc}
X_{\alpha} & \rightarrow & \operatorname{colim} X \\
\downarrow & & \downarrow \\
Y_{\alpha} & \rightarrow & \operatorname{colim} Y
\end{array}
$$

Proposition 2.6. If $\beta$ is a limit ordinal, $X, Y: \beta \rightarrow \mathcal{T}^{\mathrm{C}}$ preserve colimits, $X_{\alpha} \rightarrow X_{\alpha+1}$ and $Y_{\alpha} \rightarrow Y_{\alpha+1}$ are cofibrations for all $\alpha<\beta, f: X \rightarrow Y$ and all $f_{\alpha}$ are homotopy equivalences then colim $f$ is also a homotopy equivalence. 
By taking the mapping cylinder of $f$ we may put ourselves into the situation of Proposition 2.5. The proof then proceeds by transfinite induction.

If $J: \mathbf{C}^{\prime} \rightarrow \mathbf{C}$ is the inclusion of a closed subcategory, i.e., a subcategory with each $\mathbf{C}^{\prime}(c, d)$ closed in $\mathbf{C}(c, d)$, then a morphism $f$ in $\mathcal{J}^{\mathbf{C}}$ is a homotopy equivalence ( fibration, cofibration) rel $\mathbf{C}$ if $J^{*} f$ is a homotopy equivalence (fibration, cofibration) in $\mathcal{T}^{\mathbf{C}^{\prime}}$.

These notions are "relative" to a subcategory of the index category rather than to a subobject of the domain, as is more usual in homotopy theory. However, since we shall not need the latter sense of the word "relative" here this should lead to no confusion. The condition that the subcategory be closed, we may remark, is no real restriction; no generality would be gained by relaxing it. Absolute homotopy is subsumed under relative homotopy as the case $\mathbf{C}^{\prime}=\mathbf{C}$.

Homotopy equivalences rel $\mathbf{C}^{\prime}$ form a class which is saturated in the sense that it contains all isomorphisms and that if it contains any two of the morphisms $f, g, f g$ then it also contains the third.

We have described above the behavior of absolute homotopy equivalences, fibrations and cofibrations with respect to limits and colimits in $\mathcal{T} \mathbf{C}$ : let us describe these collectively as the limit-properties of these notions.

Proposition 2.7. If $\mathbf{C}^{\prime}$ is a closed subcategory of $\mathbf{C}$ then the homotopy equivalences, cofibrations and fibrations rel $\mathbf{C}^{\prime}$ in $\mathcal{T}^{\mathbf{C}}$ share the limit properties of the absolute ones.

This results immediately from the fact that if $J: \mathbf{C}^{\prime} \subset \mathbf{C}$ is the inclusion then $J^{*}$ preserves and reflects all limits and colimits as well as the functors $X \mapsto X \times I$, $X \mapsto X^{I}$.

3. Free prolongations; $\mathbf{C}^{\prime}$-cofibrations. The constructions we make here, as well as the proof of the "mapping-cylinder" theorem below, stem from the version of the Milgram bar construction developed by Steenrod in [12] (cf. also [5]).

If $J: \mathbf{C}^{\prime} \rightarrow \mathbf{C}$ is the inclusion of a closed subcategory and $X: \mathbf{C} \rightarrow \mathcal{T}$ we may think of $X$ as giving an "action" of $\mathbf{C}$ on its restriction $J^{*} X: \mathbf{C}^{\prime} \rightarrow \mathcal{T}$. If $J^{*} X \rightarrow A$ is a cofibration in $\mathcal{T}^{\mathbf{D}}$ we define the free prolongation along $A$, rel $\mathbf{C}^{\prime}$, of this action by the pushout

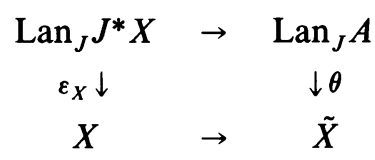

in $\mathcal{T}^{\mathbf{C}}$, where $\varepsilon$ is the counit of the adjunction $\operatorname{Lan}_{J} \rightarrow J^{*}$, whose unit we denote by $\eta$.

LEMMA 3.1. If $X \rightarrow \tilde{X}$ is a free prolongation as above then $X \rightarrow \tilde{X}$ is a cofibration in $\mathcal{T}^{\mathbf{C}}$ and $\left(J^{*} \theta\right) \eta_{A}: A \rightarrow J^{*} \tilde{X}$ is a cofibration in $\mathcal{T}^{\mathbf{C}^{\prime}}$.

The first statement follows from Proposition 2.1. For the second, observe that

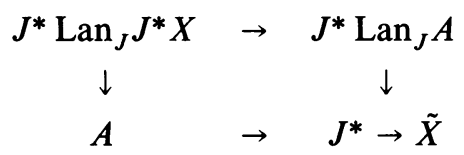

is a pushout. 
LemMA 3.2. If $\mathbf{C}^{\prime \prime} \subset \mathbf{C}^{\prime} \subset \mathbf{C}$ are closed subcategories and $X \rightarrow \tilde{X}$ in $\mathcal{T}^{\mathbf{C}}$ is a free prolongation rel $\mathbf{C}^{\prime \prime}$ then it is also a free prolongation rel $\mathbf{C}^{\prime}$.

Suppose it is a free prolongation along $\left(J J^{\prime}\right)^{*} X \rightarrow A$, where $J, J^{\prime}$ are the inclusions. Let $J^{*} X \rightarrow Y$ be the free prolongation (in $\mathcal{T}^{\mathbf{C}^{\prime}}$ ) along $A \operatorname{rel} \mathbf{C}^{\prime \prime}$. Then $X \rightarrow \tilde{X}$ is the free prolongation along $Y \operatorname{rel} \mathbf{C}^{\prime}$.

Dually if $B \rightarrow J^{*} X$ is a fibration in $\mathcal{T}^{\mathbf{C}^{\prime}}$ we define the free elevation over $B$, rel $\mathbf{C}^{\prime}$ by the pullback

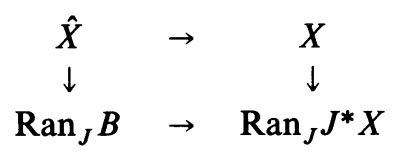

The reader may supply the duals of Lemmas 3.1, 3.2.

In order to define the notion of contraction we introduce a monoid structure in $I$, viz. the multiplication $(s, t) \rightarrow \min (s, t)$. The unit is 1 and 0 is an absorbing element. (The choice is somewhat arbitrary: The multiplication of $\mathbf{R}$, for example, could also serve. The one we have chosen is better suited to a simplicial interpretation which we shall not pursue here.) A contraction on an $X: \mathbf{C} \rightarrow \mathcal{T}$ is an action of this monoid on $X$, i.e., a morphism $I \times X \rightarrow X$ subject to the usual unit and associativity conditions. It is of course a contraction onto the subobject $X_{0}=0 \cdot X$. A retraction is an action of the submonoid $\dot{I}=\{0,1\} \subset I$; this is evidently equivalent to the usual notion. The restriction of a contraction is its associated retraction.

Of course a contraction is a homotopy and both the inclusion $X_{0} \rightarrow X$ and the retracting map 0: $X \rightarrow X_{0}$ are homotopy equivalences. If $f: X \rightarrow Y$ then the mapping cylinder $M_{f}$ of $f$ has a contraction onto $Y$ given by $s \cdot \theta(x, t)=\theta(x, \min (s, t))$.

Suppose now that $X \subset Y \subset Z$ in $\mathcal{T}^{\mathbf{C}}$, that $Y$ is provided with a contraction onto $X$ and that $Z$ has a retraction onto $X$ which extends the retraction associated to the contraction of $Y$. We construct the free prolongation of the contraction along the retraction by taking the pushout

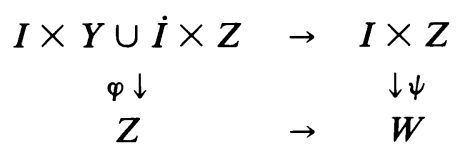

where $\varphi$ is defined by the contraction and the retraction. A contraction of $W$ onto $X$, extending both, is defined by $s \cdot \psi(t, z)=\psi(\min (s, t), z)$.

LEMMA 3.3. If $X \rightarrow Y \rightarrow Z$, as above, are cofibrations in $\mathcal{T}^{\mathbf{C}}$ then so also is $Z \rightarrow W$.

For each closed subcategory $\mathbf{C}^{\prime} \subset \mathbf{C}$ we shall distinguish, in addition to the cofibrations rel $\mathbf{C}^{\prime}$ in $\mathcal{T}^{\mathbf{C}}$, the class of $\mathbf{C}^{\prime}$-cofibrations as the smallest class containing the free prolongations rel $\mathbf{C}^{\prime}$ which is closed under right infinite composition. Equivalently, this may be described as the class of all right-infinite compositions of free prolongations.

The class of $\mathbf{C}^{\prime}$-fibrations is defined dually: it contains the free elevations rel $\mathbf{C}^{\prime}$ and is closed under left-infinite composition.

From Lemma 3.2 and its dual we may easily deduce the following statement. 
Proposition 3.4. If $\mathbf{C}^{\prime \prime} \subset \mathbf{C}^{\prime} \subset \mathbf{C}$ are closed subcategories then

$\left\{\mathbf{C}^{\prime \prime}\right.$-cofibrations $\} \subset\left\{\mathbf{C}^{\prime}\right.$-cofibrations $\} \subset\{\mathbf{C}$-cofibrations $\}=\{$ cofibrations $\}$ $=\{$ cofibrations rel $\mathbf{C}\} \subset\left\{\right.$ cofibrations rel $\left.\mathbf{C}^{\prime}\right\} \subset\left\{\right.$ cofibrations rel $\left.\mathbf{C}^{\prime \prime}\right\}$.

The corresponding statement for fibrations holds as well.

Our principal technical tool is the following relative mapping-cylinder construction, which generalizes the "absolute" mapping cylinder of $\$ 2$.

THEOREM 3.5. If $\mathbf{C}^{\prime} \subset \mathbf{C}$ is a closed subcategory then any morphism $f: X \rightarrow Y$ in $\mathcal{T} \mathbf{C}$ has a factorization $X \rightarrow \tilde{Y} \rightarrow Y$ where $X \rightarrow \tilde{Y}$ is a $\mathbf{C}^{\prime}$-cofibration and $\tilde{Y} \rightarrow Y$ is a homotopy equivalence rel $\mathbf{C}^{\prime}$, as well as a factorization $X \rightarrow \hat{X} \rightarrow Y$ where $X \rightarrow \hat{X}$ is a homotopy equivalence rel $\mathbf{C}^{\prime}$ and $\hat{X} \rightarrow Y$ a $\mathbf{C}^{\prime}$-fibration.

We shall confine ourselves to proving the first assertion, the proof of the second being a straightforward dualization.

Without loss of generality we may suppose that $f$ is a cofibration in $\mathcal{T}^{\mathbf{C}}$. Let us construct sequences of cofibrations

$$
\begin{aligned}
X & =Y_{0} \rightarrow Y_{1} \rightarrow \cdots \quad \text { in } \mathcal{T}^{\mathbf{C}}, \\
J^{*} Y & =A_{1} \rightarrow J^{*} Y_{1} \rightarrow A_{2} \rightarrow J^{*} Y_{2} \rightarrow \cdots \quad \text { in } \mathcal{T}^{\mathbf{C}^{\prime}}
\end{aligned}
$$

together with maps $r_{i}: Y_{i} \rightarrow Y$ and contractions of the $A_{i}$ onto $J^{*} Y$ according to the following prescriptions.

(0) $r_{0}=f ; A_{1}$ has the trivial contraction;

(i) each $Y_{j}, j \geqslant 1$, is the free prolongation rel $\mathbf{C}^{\prime}$ of $Y_{j-1}$ along $J^{*} Y_{j-1} \rightarrow A$, and $r_{j}$ is determined by the retraction associated to the contraction of $A_{j}$;

(ii) each $A_{j+1}$ is the free prolongation of the contraction of $A_{j}$ onto $J^{*} Y$, along $J^{*} r_{j}$, which is a retraction of $J^{*} Y_{j}$ onto $J^{*} Y$.

We set $\tilde{Y}=\operatorname{colim}\left(Y_{0} \rightarrow Y_{1} \rightarrow \cdots\right)$ so that $X \rightarrow \tilde{Y}$ is a $\mathbf{C}^{\prime}$-cofibration. But $J^{*} \tilde{Y}=$ $\operatorname{colim}\left(A_{1} \rightarrow A_{2} \rightarrow \cdots\right)$ so that, if $r: \tilde{Y} \rightarrow Y$ is the colimit of the $r_{j}$, then $J^{*} r$ is the retraction associated with the colimit of the contractions of the $A_{j}$, which of course contracts $J^{*} \tilde{Y}$ onto $J^{*} Y$.

4. Homotopy and the functor calculus. If we place ourselves in the situation of (1.2a) we may observe that, because of the adjointness asserted by (1.4), the functors $X \otimes_{\mathbf{C}^{-}}$preserve colimits. From (1.5) it follows that they preserve products with constants and in particular with $I$. These statements, together with their duals, imply the following assertions about abolute homotopy.

Proposition 4.1. (a) The functors $X \otimes_{\mathbf{C}^{-}},-\otimes_{\mathbf{C}} Y$ preserve cofibrations,

$\left(b^{\prime}\right) \operatorname{Hom}_{\mathbf{C}}(X,-)$ preserves fibrations,

$\left(b^{\prime \prime}\right) \operatorname{Hom}_{\mathbf{C}}(-, Y)$ takes cofibrations to fibrations.

Furthermore all of these functors preserve homotopy equivalences.

Corollary 4.2. If $X, Y$ are in $\mathcal{T}^{\mathbf{C}}$ then $\operatorname{Ho}^{\mathrm{T}} \mathbf{C}(X, Y)$ is canonically isomorphic to $\pi_{0} \operatorname{Hom}_{\mathbf{C}}(X, Y)$. 
Included among the functors mentioned in Proposition 4.1 are in particular the functors $F^{*}, \operatorname{Lan}_{F}$ and $\operatorname{Ran}_{F}$ associated with an $F: \mathbf{C} \rightarrow \mathbf{D}$ in $\S 1$.

Proposition 4.1(a) may be amplified by making use of a criterion for cofibrations introduced by Puppe in the classical case $\mathbf{C}=1$. If $\varphi: Y \rightarrow I$ in $\mathcal{T}^{\mathbf{C}}$ the 0 -space of $\varphi$ and its halo are the pullbacks:

$$
\begin{array}{cccccc}
X & \rightarrow & Y & U & \rightarrow & Y \\
\downarrow & & \downarrow \varphi & \downarrow & & \downarrow \varphi \\
\{0\} & \rightarrow & I & {[0,1)} & \rightarrow & I
\end{array}
$$

The proof of the following lemma proceeds exactly as in the classical case, as does its indicated consequence.

LEMMA 4.3. A morphism $X \rightarrow Y$ in $\mathcal{T}^{\mathbf{C}}$ is a cofibration if and only if there is a $\varphi$ : $Y \rightarrow I$ whose 0-space is $X \rightarrow Y$, and a homotopy, stationary on $X$, of the identity of $Y$ with a morphism taking the halo into $X$.

Proposition 4.4. In the situation of (1.2a) suppose that $X^{\prime} \rightarrow X$ and $Y^{\prime} \rightarrow Y$ are cofibrations. Then

$$
\begin{array}{ccc}
X^{\prime} \otimes_{\mathbf{C}} Y^{\prime} & \rightarrow & X \otimes_{\mathbf{C}} Y^{\prime} \\
\downarrow & & \downarrow \\
X^{\prime} \otimes_{\mathbf{C}} Y & \rightarrow & X \otimes_{\mathbf{C}} Y
\end{array}
$$

is a 2-cofibration.

The behavior of relative homotopy with respect to the functor calculus is governed by the following lemma.

Lemma 4.5. Let $\mathbf{B}^{\prime} \subset \mathbf{B}, \mathbf{C}^{\prime} \subset \mathbf{C}, \mathbf{D}^{\prime} \subset \mathbf{D}$ be closed subcategories.

(a) If $f: X \rightarrow X^{\prime}$ is a $\mathbf{B} \times \mathbf{C}^{\prime \mathrm{op}_{-}}$-cofibration in $\boldsymbol{\sigma}^{\mathbf{B} \times \mathbf{C}^{\mathrm{op}}}, \mathrm{g}$ is a homotopy equivalence rel $\mathbf{C}^{\prime} \times \mathbf{D}^{\prime}$ in $\mathcal{T} \mathbf{C} \times \mathbf{D}$ and $X \otimes_{\mathbf{C}} g$ is a homotopy equivalence rel $\mathbf{B} \times \mathbf{D}^{\prime}$ then so also is $X^{\prime} \otimes_{\mathrm{C}} g$.

(b) If $f: X \rightarrow X^{\prime}$ is a $\mathbf{B} \times \mathbf{C}^{\prime}$-cofibration in $\mathcal{T}^{\mathbf{B} \times \mathbf{C}}, g$ is a homotopy equivalence rel $\mathbf{C}^{\prime} \times \mathbf{D}^{\prime}$ in $\mathcal{T} \mathbf{C}^{\mathbf{C}}$ and $\operatorname{Hom}_{\mathbf{C}}(X, g)$ is a homotopy equivalence rel $\mathbf{B}^{\mathrm{op}} \times D^{\prime}$ then so also is $\operatorname{Hom}_{\mathbf{C}}\left(X^{\prime}, g\right)$.

$\left(\mathrm{b}^{\prime \prime}\right)$ If $f$ is a homotopy equivalence rel $\mathbf{B}^{\prime} \times \mathbf{C}^{\prime}$ in $\mathcal{T}^{\mathbf{B} \times \mathbf{C}}$, g: $Y^{\prime} \rightarrow Y$ is a $\mathbf{C}^{\prime} \times \mathbf{D}$ fibration in $\mathcal{G} \mathbf{C} \times \mathbf{D}$ and $\operatorname{Hom}_{\mathbf{C}}(f, Y)$ is a homotopy equivalence rel $\mathbf{B}^{\prime o p} \times \mathbf{D}$ then so also is $\operatorname{Hom}_{\mathbf{C}}\left(f, Y^{\prime}\right)$.

These statements - and their proofs - are related to one another by dualization; it will be sufficient to verify the first one. Moreover, restriction to $\mathbf{D}^{\prime} \subset \mathbf{D}$ commutes with the tensor product over $\mathbf{C}$, so that we may assume without loss of generality that $\mathbf{D}^{\prime}=\mathbf{D}$.

Let us suppose to begin with, that $f: X \rightarrow X^{\prime}$ is a free prolongation rel $\mathbf{B} \times \mathbf{C}^{\prime o p}$ along, say, $\left(\mathbf{B} \times J^{*}\right) X \rightarrow A$ where $J: \mathbf{C}^{\prime} \subset \mathbf{C}$ is the inclusion. If $g: Y \rightarrow Y^{\prime}$ we may 
then consider the diagram:

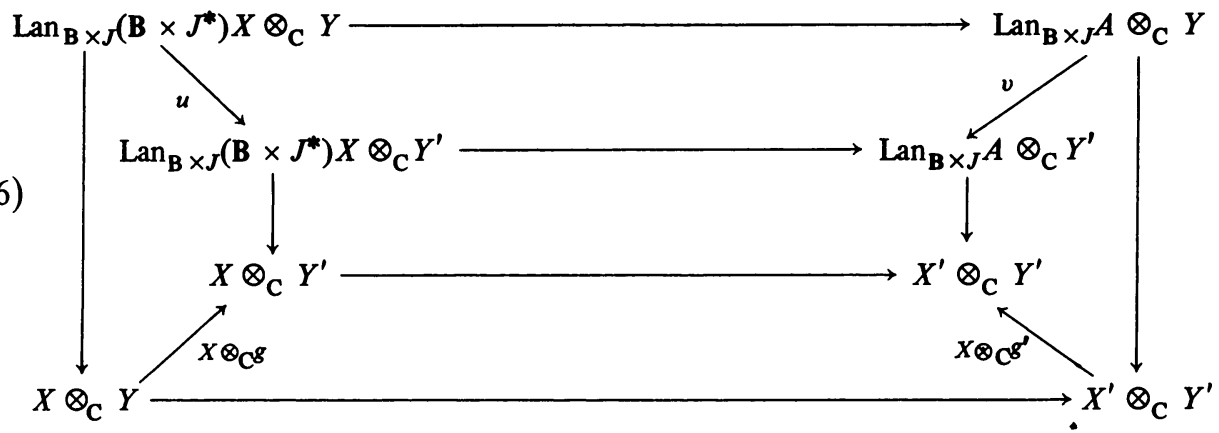

The inner and outer squares are pushouts and the horizontal arrows are cofibrations. Furthermore $v=\operatorname{Lan}_{\mathbf{B} \times J} A \otimes_{\mathbf{C}} g=A \otimes_{\mathbf{C}^{\prime}}\left(J^{*} \times \mathrm{D}\right) g$. Thus $v$, and similarly $u$, are homotopy equivalences. The conclusion now follows from Proposition 2.1(iii).

The general case, with $f$ any $\mathbf{B} \times \mathbf{C}^{\text {op }}$-cofibration follows, using Proposition 2.6.

If $\varnothing \rightarrow X$ is a $\mathbf{C}^{\prime}$-cofibration in $\mathcal{T}^{\mathbf{C}}$ ( $Y \rightarrow *$ is a $\mathbf{C}^{\prime}$-fibration) then $X$ is cofibrant ( $Y$ is fibrant $)$. The functors, $X \otimes_{\mathbf{C}^{-}}, \operatorname{Hom}_{\mathbf{C}}(X,-), \operatorname{Hom}_{\left.\mathbf{C}^{(-}, Y\right)}$ in these cases preserve suitable relative homotopy equivalence. With the help of Lemma 4.5 and Proposition 2.3 we get the following result.

Proposition 4.7. If $X$ is $\mathbf{C}^{\prime}$-cofibrant ( $Y$ is $\mathbf{C}^{\prime}$-fibrant) in $\mathcal{T}^{\mathbf{C}}$ then $\mathrm{Ho} \mathcal{T}^{\mathbf{C}}(X,-)$

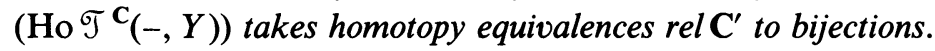

This will allow us to conclude the existence of categories of fractions with respect to relative homotopy equivalences. We shall extend our terminology by defining, for a closed subcategory $\mathbf{C}^{\prime} \subset \mathbf{C}$, the class of homotopy equivalences rel $\mathbf{C}$ in $\mathrm{Ho}^{\mathbf{C}}$ as the smallest saturated class containing the images of the ones in $\widetilde{T} \mathbf{C}$.

THEOREM 4.8. The class of homotopy equivalences rel $\mathbf{C}^{\prime}$ in Ho $\mathcal{T}^{\mathbf{C}}$ admits both a calculus of left fractions and a calculus of right fractions. The corresponding category of fractions $\mathrm{Ho}_{\mathbf{C}^{\prime}} \sigma^{\mathrm{T}}$ is also a category of fractions with respect to the homotopy equivalences rel $\mathbf{C}^{\prime}$ in $\mathcal{T}^{\mathbf{C}}$.

This follows at once from Propositions 2.1 and 2.7, Theorem 3.5 and Proposition 4.7. We may note in particular that if $X$ is $\mathbf{C}^{\prime}$-cofibrant or $Y$ is $\mathbf{C}^{\prime}$-fibrant then

$$
\mathrm{Ho}_{\mathbf{C}^{\prime}} \sigma^{\mathbf{c}}(X, Y) \approx \operatorname{Ho}{ }^{\mathbf{c}}(X, Y) .
$$

If $\mathbf{C}^{\prime}=\mathbf{C}$ then of course $\mathrm{Ho}_{\mathbf{C}^{\prime}} \mathcal{T}^{\mathbf{C}}=\mathrm{Ho} \mathcal{T}^{\mathbf{C}}$. We may abbreviate $\mathrm{Ho}_{\mathbf{C}_{0}} \mathcal{T}^{\mathbf{C}}$ to $\mathrm{Ho}_{0} \sigma^{\mathbf{C}}$.

5. Some adjoint functors between homotopy categories. Let us begin by recording a familiar fact about categories of fractions.

Proposition 5.1. Let $a: \mathbb{Q} \rightarrow \mathscr{Q}\left[S^{-1}\right]$ and $b: \mathscr{B} \rightarrow \mathscr{B}\left[T^{-1}\right]$ be categories of fractions. If $F: \mathbb{Q} \rightarrow \mathscr{B}$ takes $S$ into $T$ then there is a unique $\bar{F}: \mathscr{Q}\left[S^{-1}\right] \rightarrow \mathscr{B}\left[T^{-1}\right]$ such that $\bar{F} a=b F$. If also $G: \mathscr{B} \rightarrow \mathbb{Q}$ takes $T$ into $S$ and $F+G$ then $\bar{F}+\bar{G}$. 
For the latter statement it is only necessary to observe that the unit and counit of the adjunction $F+G$ provide those for $\bar{F}+\bar{G}$.

We may apply this as follows.

Proposition 5.2. Suppose that $\mathbf{C}^{\prime} \subset \mathbf{C}$ and $\mathbf{D}^{\prime} \subset \mathbf{D}$ are closed subcategories and that $X: \mathbf{C}^{\mathrm{op}} \times \mathbf{D} \rightarrow$ T. Then

(i) If $\operatorname{Hom}_{\mathbf{D}}(X,-): \mathcal{T}^{\mathbf{D}} \rightarrow \mathcal{T}^{\mathbf{C}}$ takes homotopy equivalences rel $\mathbf{D}^{\prime}$ to homotopy equivalences rel $\mathbf{C}^{\prime}$ then the induced functor $R: \mathrm{Ho}_{\mathbf{D}^{\prime}} \mathcal{T}^{\mathbf{D}} \rightarrow \mathrm{Ho}_{\mathbf{C}^{\prime}} \mathcal{T}^{\mathbf{C}}$ has a left adjoint.

(ii) If $X \otimes_{\mathbf{C}^{-}}: \mathcal{T}^{\mathbf{C}} \rightarrow \mathcal{T}^{\mathbf{D}}$ takes homotopy equivalences rel $\mathbf{C}^{\prime}$ to homotopy equivalences rel $\mathbf{D}^{\prime}$ then the induced functor $L: \mathrm{Ho}_{\mathbf{C}^{\prime}} \mathcal{T}^{\mathbf{C}} \rightarrow \mathrm{Ho}_{\mathbf{D}^{\prime}} \mathcal{T}^{\mathrm{D}}$ has a right adjoint.

It will be sufficient to prove (i). Let $\tilde{X} \rightarrow X$ be a homotopy equivalence rel $\mathbf{C}^{\prime \text { op }} \times \mathbf{D}$ such that $\tilde{X}$ is $\mathbf{C}^{\mathrm{op}} \times \mathbf{D}$-cofibrant. Then, by Proposition 4.1, $\operatorname{Hom}_{\mathbf{D}}(X, Y) \rightarrow$ $\operatorname{Hom}_{\mathrm{D}}(\tilde{X}, Y)$ is for all $Y$ a homotopy equivalence rel $\mathbf{C}^{\prime}$ and, up to isomorphism, $\operatorname{Hom}_{\mathrm{D}}(\tilde{X},-)$ induces the same functor on the homotopy categories as does $\operatorname{Hom}_{\mathbf{D}}(X,-)$. But $\tilde{X} \otimes_{\mathbf{C}^{-}}$is left adjoint to $\operatorname{Hom}_{\mathbf{D}}(\tilde{X},-1)$ and, by Lemma 4.5 , takes homotopy equivalences rel $\mathbf{C}^{\prime}$ to homotopy equivalences in $\mathcal{T}^{\mathbf{D}}$, a fortiori to homotopy equivalences rel $\mathbf{D}^{\prime}$. The conclusion now follows from Proposition 5.1.

A special case is worthy of particular note. If $\mathbf{C}^{\prime} \subset \mathbf{C}$ and $\mathbf{D}^{\prime} \subset \mathbf{D}$ are closed subcategories and $F: \mathbf{C} \rightarrow \mathbf{D}$ takes $\mathbf{C}^{\prime}$ into $\mathbf{D}^{\prime}$ - we shall write $F:\left(C, \mathbf{C}^{\prime}\right) \rightarrow\left(\mathbf{D}, \mathbf{D}^{\prime}\right)$ then $F^{*}: \mathcal{T}^{\mathbf{D}} \rightarrow \mathcal{T}^{\mathbf{C}}$ clearly takes homotopy equivalences rel $\mathbf{D}^{\prime}$ to homotopy equivalences rel $\mathbf{C}^{\prime}$ and thus induces on the homotopy categories $\mathrm{Ho}_{\mathbf{C}^{\prime}, \mathbf{D}^{\prime}} \mathrm{F}^{*}: \mathrm{Ho}_{\mathbf{D}^{\prime}} \mathcal{T}^{\mathbf{D}} \rightarrow$ $\mathrm{Ho}_{\mathbf{C}^{\prime}} \mathcal{T}^{\mathbf{C}}$. Thus (1.6) and Proposition 5.2 imply the following statement.

THeOREM 5.3. If $F:\left(\mathbf{C}, \mathbf{C}^{\prime}\right) \rightarrow\left(\mathbf{D}, \mathbf{D}^{\prime}\right)$ then $\mathrm{Ho}_{\mathbf{C}^{\prime}, \mathbf{D}^{\prime}} F^{*}$ has adjoints on both sides.

We denote these by

$$
\mathrm{Ho}_{\mathbf{C}^{\prime}, \mathbf{D}^{\prime}} \operatorname{lan}_{F}+\mathrm{Ho}_{\mathbf{C}^{\prime}, \mathbf{D}^{\prime}} F^{*}+\mathrm{Ho}_{\mathbf{C}^{\prime}, \mathbf{D}^{\prime}}, \operatorname{ran}_{F} .
$$

They are the left and right homotopy Kan extensions along $F$, relative to $\mathbf{C}^{\prime}, \mathbf{D}^{\prime}$.

In the special case $\mathbf{C}^{\prime}=\mathbf{C}, \mathbf{D}^{\prime}=\mathbf{D}$ we may omit the subscripts. If $\mathbf{C}^{\prime}=\mathbf{C}_{0}$, $\mathbf{D}^{\prime}=\mathbf{D}_{0}$ we abbreviate to $\mathrm{Ho}_{0} \operatorname{lan}_{F}, \mathrm{Ho}_{0} F^{*}, \mathrm{Ho}_{0} \mathrm{ran}_{F}$.

A still more special case has its own notation. If $F: \mathbf{C} \times \mathbf{D} \rightarrow \mathbf{D}$ is the projection then the left and right homotopy Kan extensions along $F$ are the homotopy colimit and the homotopy limit. If $\mathbf{C}^{\prime} \subset \mathbf{C}, \mathbf{D}^{\prime} \subset \mathbf{D}$ we write $\mathrm{Ho}_{\mathbf{C}^{\prime}, \mathbf{D}^{\prime}}$ colim $\mathbf{C}=\mathrm{Ho}_{\mathbf{C}^{\prime} \times \mathbf{D}^{\prime}, \mathbf{D}^{\prime}} \operatorname{lan}_{F}$ and so forth. The case $\mathbf{D}=\mathbf{1}$ is still nontrivial: it gives the "classical" homotopy limit and colimit.

These homotopy Kan extensions may be expressed by formulae. Let $\tilde{D} \rightarrow \mathbf{D}$ 。 $(F \times 1)$ be a homotopy equivalence rel $\mathbf{C}^{\prime \text { op }} \times \mathbf{D}$ such that $\tilde{D}$ is $\mathbf{C}^{\prime \mathrm{op}} \times \mathbf{D}$-cofibrant. Then $\mathrm{Ho}_{\mathbf{C}^{\prime}, \mathbf{D}^{\prime}} \operatorname{lan}_{F} X \approx \tilde{D} \otimes_{\mathbf{C}} X$. If $\hat{D} \rightarrow \mathbf{D} \circ(1 \times F)$ has the corresponding properties, then

$$
\mathrm{Ho}_{\mathbf{C}^{\prime}, \mathbf{D}^{\prime}} \operatorname{ran}_{F} X \approx \operatorname{Hom}_{\mathbf{C}}(\hat{D}, X) .
$$

An easy extension of the argument of Proposition 5.2 leads also to the following conclusion.

Proposition 5.4. If $X$ is $\mathbf{C}^{\prime}$-cofibrant then $\operatorname{Ho}_{\mathbf{C}^{\prime}, \mathbf{D}^{\prime}} \operatorname{lan}_{F} X \approx \operatorname{Lan}_{F} X$; if $X$ is

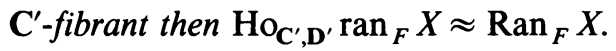


Analogous statements for homotopy limits and colimits constitute a special case.

We may recall here the observation of $R$. Vogt [13] that if $\mathbf{C}$ is a monoid in $\mathcal{T}$ then $\mathrm{Ho}_{0} \lim _{\mathbf{C}}(*)$ is (up to homotopy) the Milgram bar-construction $B C$. Indeed the same statement is true for any $\mathcal{T}_{\text {-category }} \mathbf{C}$, if $B C$ is taken to be the Segal classifying space of $\mathbf{C}$ (cf. [11]). The argument, which depends on a careful examination of these constructions, is not germane to our present interests and will be omitted.

It is a familiar observation that if $F: \mathbf{C} \rightarrow \mathbf{D}$ is a morphism of topological monoids which is a homotopy equivalence on the underlying spaces then $B F: B C \rightarrow B D$ is a homotopy equivalence as well. We may generalize this result in the following way.

Let us say that a functor $F: \mathbf{C} \rightarrow \mathbf{D}$ is an $H$-equivalence of $\mathcal{T}$-categories if $F_{0}$ : $\mathbf{C}_{0} \approx \mathbf{D}_{0}$ and, for each $c_{0}, c_{1}, \mathbf{C}\left(c_{0}, c_{1}\right) \rightarrow \mathbf{D}\left(c_{0}, c_{1}\right)$ is a homotopy equivalence.

THEOREM 5.5. If F: $\mathbf{C} \rightarrow \mathbf{D}$ is an H-equivalence then $\mathrm{Ho}_{0} F^{*}: \mathrm{Ho}_{0} \mathcal{T}^{\mathbf{D}} \rightarrow \mathrm{Ho}_{0} \mathcal{T}^{\mathbf{C}}$ is an equivalence of categories.

It will follow that $\mathrm{Ho}_{0} \operatorname{lan}_{F}, \mathrm{Ho}_{0} \operatorname{ran}_{F}$ are both, up to isomorphism, inverses of $\mathrm{Ho}_{0} F^{*}$.

Without loss of generality we may assume that $\mathbf{C}_{0}=\mathbf{D}_{0}$ and that $F_{0}$ is the identity. By Proposition 5.4 if $X$ is $\mathbf{C}_{0}$-cofibrant in $\mathcal{T}^{\mathbf{C}}$ then $\mathrm{Ho}_{0} \operatorname{lan}_{F} X \approx \operatorname{Lan}_{F} X \approx \mathrm{D} \circ(F$ $\times 1) \otimes_{\mathbf{C}} X$ and $\left(\mathrm{Ho}_{0} F^{*}\right)\left(\mathrm{Ho}_{0} \operatorname{lan}_{F}\right) X \approx \mathbf{D} \circ(F \times F) \otimes_{\mathbf{C}} X$. Now the morphism $\mathbf{C}$ $\rightarrow \mathbf{D} \circ(F \times F)$ in $\mathcal{T}^{\mathbf{C}^{\mathrm{op}} \times \mathbf{C}}$ defined by $F$ is by our hypothesis a homotopy equivalence rel $\mathrm{C}_{0} \times \mathrm{C}_{0}$. The unit $\eta_{X}: X \rightarrow\left(\mathrm{Ho}_{0} F^{*}\right)\left(\mathrm{Ho}_{0} \operatorname{lan}_{F}\right) X$ of the adjunction is represented by $X \approx \mathbf{C} \otimes_{\mathbf{C}} X \rightarrow \mathbf{D} \circ(F \times F) \otimes_{\mathbf{C}} X$ which by Lemma 4.5 is a homotopy equivalence rel $\mathbf{C}_{0}$. Since any $X$ in $\mathcal{T}^{\mathbf{C}}$ is homotopy-equivalent rel $\mathbf{C}_{0}$ to a $\mathbf{C}_{0}$-cofibrant object this implies that all $\eta_{X}$ are isomorphisms.

On the other hand $F^{*}$ reflects homotopy equivalences rel $\mathrm{C}_{0}$; it follows at once that for all $Y$ in $\mathcal{T}^{\mathbf{D}}$ the counit $\varepsilon_{Y}:\left(\mathrm{Ho}_{0} \operatorname{lan}_{F}\right)\left(\mathrm{Ho}_{0} F^{*}\right) Y \rightarrow Y$ is also an isomorphism.

6. Some special cases. In some cases homotopy limits and colimits can be described more simply than by the constructions of $\S 5$. The categories $\mathrm{Ho}_{\mathbf{C}^{\prime}} \mathcal{T}^{\mathbf{C}}$ have of course products and coproducts, coming from those of $\mathcal{J}^{\mathbf{c}}$, and these are easily seen to be homotopy products and coproducts as well. We shall consider here some homotopy colimits over ordered sets, specifically, homotopy pushouts and ordinal homotopy colimits. For brevity we shall, here, denote a functor $\Lambda \rightarrow \mathcal{T}^{\mathbf{C}}$ and its transpose $\Lambda \times \mathbf{C} \rightarrow \mathcal{T}$ by the same letter.

LEMMA 6.1. Suppose that $X \rightarrow \tilde{X}$ is a free prolongation rel $\mathbf{C} \times \Lambda_{0}$ in $\mathcal{\sigma} \mathbf{C} \times \Lambda$, where $\Lambda$ is an ordered set. If $X$ has the property that $X_{\lambda} \rightarrow X_{\mu}$ is a cofibration for all $\lambda \leqslant \mu$ then also

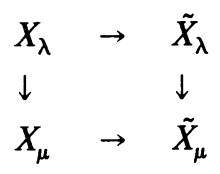

is a 2-cofibration. 
Let $J: \mathbf{C} \times \Lambda_{0} \rightarrow \mathbf{C} \times \Lambda$ be the inclusion. For any $A: \mathbf{C} \times \Lambda_{0} \rightarrow \mathcal{T},\left(\operatorname{Lan}_{J} A\right)_{\mu}=$ $\mathrm{U}_{\gamma<\mu} A_{\gamma}$. If $X \rightarrow \tilde{X}$ is a free prolongation along $A$ we may construct the diagram

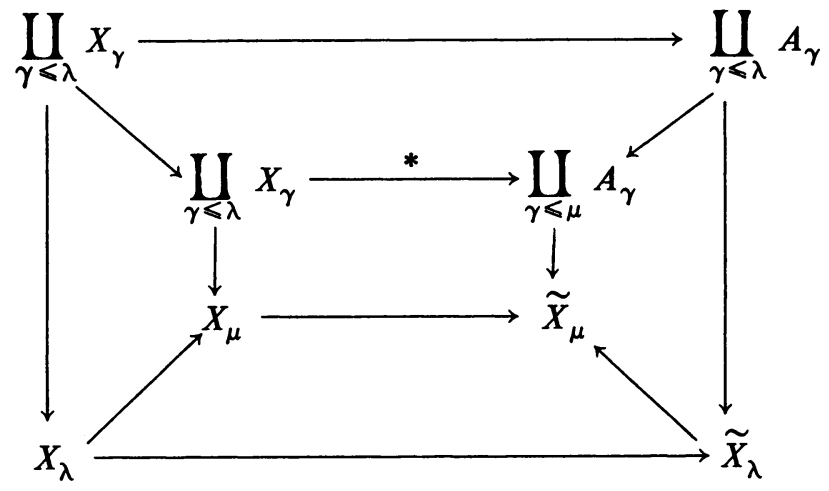

The inside and outside squares are pushouts, while the pushout in the starred square is $\amalg_{\gamma<\lambda} A_{\gamma} \sqcup \amalg_{\gamma<\mu, \gamma \neq \lambda} X_{\gamma}$. Thus the starred square is a 2-cofibration and the conclusion follows from Proposition 2.3. Using Proposition 2.5 we may deduce the following statement.

COROllary 6.2. If $X$ is $\mathbf{C} \times \Lambda_{0}$-cofibrant in $\mathfrak{T}{ }^{\mathbf{C} \times \Lambda}$ then $X_{\lambda} \rightarrow X_{\mu}$ is a cofibration for any $\lambda \leqslant \mu$ in $\Lambda$.

Pushouts are colimits over the category $\mathbf{W}=(1 \leftarrow 0 \rightarrow 2)$. Homotopy pushouts may be identified in the following way.

Proposition 6.3. If $X: \mathbf{C} \times \mathbf{W} \rightarrow \mathcal{T}$ and $X_{0} \rightarrow X_{1}, X_{0} \rightarrow X_{2}$ are cofibrations rel $\mathbf{C}^{\prime}$ in $\sigma^{\mathbf{C}}$ then $\mathrm{Ho}_{\mathbf{C}^{\prime}, 0} \operatorname{colim}_{\mathbf{W}} X \approx \operatorname{colim}_{\mathbf{W}} X$.

In other words for such $X$ the pushout in $\mathcal{T}^{\mathbf{C}}$ represents the homotopy pushout in $\mathrm{Ho}_{\mathbf{C}^{\prime}} \mathcal{T}^{\mathbf{C}}$. To see that this is so, choose (using Theorem 3.5) a homotopy equivalence $\tilde{X} \rightarrow X \operatorname{rel} \mathbf{C}^{\prime} \times \mathbf{W}_{0}$ such that $\tilde{X}$ is $\mathbf{C}^{\prime} \times \mathbf{W}_{0}$-cofibrant. Now $\tilde{X}$ is also $\mathbf{C} \times \mathbf{W}_{0}$-cofibrant and, by Corollary $6.2, \tilde{X}_{0} \rightarrow \tilde{X}_{1}, \tilde{X}_{0} \rightarrow \tilde{X}_{2}$ are cofibrations in $\mathcal{T}$. It follows from Propositions 2.1 and 2.7 that $\operatorname{colim}_{\mathrm{w}} \tilde{X} \rightarrow \operatorname{colim}_{\mathbf{w}} X$ is a homotopy equivalence rel $\mathbf{C}^{\prime}$. But, in consequence of Proposition 5.4, $\mathrm{Ho}_{\mathbf{C}^{\prime}} \operatorname{colim}_{\mathbf{W}} \tilde{X} \approx \operatorname{colim}_{\mathbf{W}} \tilde{X}$.

An analogous argument proves the following assertion.

Proposition 6.4. If $\beta$ is a limit ordinal, $X: \beta \rightarrow \mathcal{T}^{\mathbf{C}}$ preserves colimits and, for all $\alpha<\beta, X_{\alpha} \rightarrow X_{\alpha+1}$ is a cofibration rel $\mathbf{C}^{\prime}$ then $\mathrm{Ho}_{\mathbf{C}^{\prime}} \operatorname{colim}_{\beta} X \approx \operatorname{colim}_{\beta} X$.

Although the categories $\mathrm{Ho}_{\mathbf{C}^{\prime}} \mathcal{T}^{\mathbf{C}}$ in general lack colimits, they have weak colimits, about which we can adduce some additional information.

Let us begin by observing that a diagram $T: \mathbf{W} \rightarrow \mathrm{Ho}_{\mathbf{C}^{\prime}} \mathcal{T}^{\mathbf{C}}$ can always be lifted to an $X: \mathbf{W} \rightarrow \mathcal{T}^{\mathbf{C}}$ with $h X \approx T$, where $h: \mathcal{T}^{\mathbf{C}} \rightarrow \mathrm{Ho}_{\mathbf{C}^{\prime}} \mathcal{T}^{\mathbf{C}}$ is the canonical functor. Thus $T$ has a colimit cone, provided by $\mathrm{Ho}_{\mathbf{C}^{\prime}, 0}$ colim $\mathbf{W}$. We shall refer to such colimit cones as pseudopushouts.

Indeed we may even suppose that the lifting $X$ is $\mathbf{C}^{\prime} \times \mathbf{W}_{0}$-cofibrant in $\mathcal{T}^{\mathbf{C} \times \mathbf{w}}$. By (4.9) this implies that, for any $Y: \mathbf{C} \times \mathbf{W} \rightarrow \mathcal{T}, \mathcal{T}^{\mathbf{C} \times \mathbf{W}}(X, Y) \rightarrow\left(\mathrm{Ho}_{\mathbf{C}^{\prime}} \mathcal{T}^{\mathbf{C}}\right)^{\mathbf{W}}(h X, h Y)$ 
is surjective. Thus if $T \rightarrow S$ in $\left(\mathrm{Ho}_{\mathbf{C}^{\prime}} \sigma^{\mathbf{C}}\right)^{\mathbf{w}}$ and $\bar{T}, \bar{S}$ are pseudopushouts these constructions give rise to morphisms $\bar{T} \rightarrow \bar{S}$ of colimit cones. Let us distinguish these as the admissible morphisms associated with $T \rightarrow S$.

Proposition 6.5. (i) Any pseudopushout in $\mathrm{Ho}_{\mathbf{C}^{\prime}} \sigma^{\mathbf{C}}$ is a weak pushout.

(ii) Any admissible morphism associated with an isomorphism is again an isomorphism.

In particular, weak pushouts always exist in $\mathrm{Ho}_{\mathbf{C}^{\prime}} \mathcal{T}^{\mathbf{C}}$, and thus, by a standard argument, all diagrams have weak colimits. The pseudopushouts which provide these are, moreover, unique up to a noncanonical isomorphism.

We have also an analogous treatment of functors $T: \beta \rightarrow \mathrm{Ho}_{\mathbf{C}^{\prime}} \mathcal{T}^{\mathbf{C}}$, where $\beta$ is a limit ordinal, if we agree to mean by a lifting a functor $X: \beta \rightarrow \mathcal{J}^{\mathbf{C}}$ such that $h X s \approx T$, when $s$ is the successor function on $\beta, s \alpha=\alpha+1$. One proves by induction on $\beta$ that any $T$ has a lifting $X$ which is colimit preserving and has the property that each $X_{\alpha} \rightarrow X_{\alpha+1}$ is a cofibration. Pseudocolimits of functors $T: \beta \rightarrow$ $\mathrm{Ho}_{\mathbf{C}^{\prime}} \mathcal{T}^{\mathbf{C}}$ are defined as the homotopy colimits of such liftings, as are the morphisms of pseudocolimits associated to morphisms $T \rightarrow S$ of such functors. These pseudocolimits are, once again, weak colimits and all morphisms associated with isomorphisms are themselves isomorphisms, so that pseudocolimits are unique up to noncanonical isomorphism.

They enjoy also the following remarkable property.

Proposition 6.6. For any $X$ in $\mathcal{T}^{\mathbf{c}}$ there is a cardinal number $\beta_{X}$ with the following property: if $\beta$ is any regular cardinal larger than $\beta_{X}, T$ is any functor $\beta \rightarrow \mathrm{Ho}_{\mathbf{C}^{\prime}} \sigma^{\mathbf{C}}$ and $\bar{T}$ is a pseudocolimit of $T$ then the canonical map

$$
\operatorname{colim}_{\alpha<\beta} \operatorname{Ho}_{\mathbf{C}^{\prime}} \mathcal{T}^{\mathbf{C}}\left(X, T_{\alpha}\right) \rightarrow \mathrm{Ho}_{\mathbf{C}^{\prime}} \mathcal{T}^{\mathbf{C}}(X, \bar{T})
$$

is bijective.

Without loss of generality we may assume that $X$ is $\mathbf{C}$-cofibrant in $\mathcal{G} \mathbf{C}$; we take $\beta_{X}$ larger than the number of points in any $X_{c} \times I, c \in \mathbf{C}_{0}$. We may further assume that $F \approx \operatorname{colim}_{\beta} Y$ where $Y: \beta \rightarrow \mathcal{T}^{\mathbf{C}}$ is colimit preserving and each $Y_{\alpha} \rightarrow Y_{\alpha+1}$ is a cofibration. But then any $X \rightarrow \bar{T}, X \times I \rightarrow \bar{T}$ both factor through some $Y_{\alpha}, \alpha<\beta$.

7. CW spaces; weak homotopy. Let us denote by $\mathcal{C}$ the full subcategory of $\mathfrak{T}$ containing the spaces of the homotopy type of a $\mathrm{CW}$ complex. $\mathcal{C}$ is neither complete nor cocomplete. It is however closed under finite products, all coproducts, $c$-pushouts and certain ordinal colimits: if $\beta$ is a limit ordinal, $X: \beta \rightarrow \mathcal{C}$ is colimit preserving,

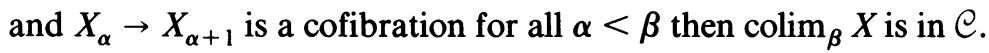

A map $f$ in $T$ is a weak homotopy equivalence if $\operatorname{Ho} \mathscr{T}(X, f)$ is bijective for all $X$ in $\mathcal{C}$. The class of weak homotopy equivalences has closure properties analogous to those of the class of homotopy equivalences (cf. §2). In a category $\mathcal{J}^{\mathbf{C}}$ we shall say that a morphism $f$ is a weak homotopy equivalence if $f_{c}$ is one, for all $c \in \mathbf{C}_{0}$. These form, evidently, a class still larger than that of the homotopy equivalences rel $\mathbf{C}_{0}$; within the category $e^{\mathbf{c}} \subset \mathcal{J}^{\mathbf{C}}$, on the other hand, they coincide.

The images in Ho $\mathcal{J}^{\mathbf{C}}$ of the weak homotopy equivalences in $\mathcal{T}^{\mathbf{C}}$ generate a saturated class of morphisms whose elements we again refer to as weak homotopy 
equivalences. We shall see that $\mathrm{Ho}_{0} \sigma^{\mathbf{C}}$ admits a calculus of left fractions with respect to this class, leading to a category of fractions $\mathrm{Ho}_{w} \mathcal{T}$, which is of course also the category of fractions of $\mathcal{T}^{\mathbf{C}}$ with respect to its weak homotopy equivalences.

If $J: \mathbf{C}_{0} \rightarrow \mathbf{C}$ is the inclusion and $A: \mathbf{C}_{0} \rightarrow \mathcal{T}$ then $\left(\operatorname{Lan}_{J} A\right)_{c}=\amalg_{c^{\prime}} \mathbf{C}\left(c^{\prime}, c\right) \times A_{c^{\prime}}$. If also $X: \mathbf{C}^{\text {op }} \rightarrow T$ then

$$
X \otimes_{\mathrm{C}} \operatorname{Lan}_{J} A \approx\left(J^{*} X\right) \otimes_{\mathrm{C}_{0}} A=\amalg_{c} X_{c} \times A_{c} .
$$

From this we may, along the lines of Proposition 4.1(a), deduce the following result.

Proposition 7.1. (i) If $X: \mathbf{C} \rightarrow \mathcal{T}$ is $\mathbf{C}_{0}$-cofibrant then $-\otimes_{\mathbf{C}} X$ preserves weak homotopy equivalences.

(ii) If $F: \mathbf{C} \rightarrow \mathbf{D}$ then $\mathrm{Ho}_{0} F^{*}$ and $\mathrm{Ho}_{0} \operatorname{lan}_{F}$ preserve weak homotopy equivalences.

We may also extend Theorem 5.5. We say that $F: \mathbf{C} \rightarrow \mathbf{D}$ is a weak H-equivalence if $F_{0}: \mathbf{C}_{0} \approx \mathbf{D}_{0}$ and each $\mathbf{C}\left(c, c^{\prime}\right) \rightarrow \mathbf{D}\left(F_{c}, F_{c^{\prime}}\right)$ is a weak homotopy equivalence.

LEMMA 7.2. If $F: \mathbf{C} \rightarrow \mathbf{D}$ is a weak $H$-equivalence then for any $X$ in $\mathcal{T}^{\mathbf{C}}, Y$ in $\mathcal{T}^{\mathbf{D}}$ the unit $\eta_{X}$ and the counit $\varepsilon_{Y}$ of the adjunction $\mathrm{Ho}_{0} \operatorname{lan}_{F} \rightarrow \mathrm{Ho}_{0} F^{*}$ are weak homotopy equivalences.

We may without loss of generality assume that $\mathbf{C}_{0}=\mathbf{D}_{0}$ with $F_{0}$ the identity and that $X$ is $\mathbf{C}_{0}$-cofibrant. Then, by Proposition 5.4, $\operatorname{Ho}_{0} \operatorname{lan}_{F} X \approx \mathbf{D} \circ(F \times 1) \otimes_{\mathbf{C}} X$ and $\left(\mathrm{Ho}_{0} F^{*}\right)\left(\mathrm{Ho}_{0} \operatorname{lan}_{F}\right) X \approx \mathrm{D} \circ(F \times F) \otimes_{\mathbf{C}} X$. The unit $\eta_{X}$ is thus given by

$$
X \approx \mathbf{C} \otimes_{\mathbf{C}} X \stackrel{\hat{F} \otimes_{\mathbf{C}} X}{\rightarrow} \mathrm{D} \circ(F \times F) \otimes_{\mathbf{C}} X
$$

where $\hat{F}: \mathbf{C} \rightarrow \mathbf{D} \circ(F \times F)$ in $\mathcal{T}^{\mathbf{C o p}^{\text {o }} \times \mathbf{C}}$ is defined by $F$ and is thus a weak homotopy equivalence. On the other hand, $\mathrm{Ho}_{0} F^{*}$ evidently reflects weak homotopy equivalences. Since $\left(\mathrm{Ho}_{0} F^{*} \varepsilon_{Y}\right)\left(\eta_{F^{*} Y}\right)=1$ it follows that $\varepsilon_{Y}$ is also a weak homotopy equivalence.

Let us say that a $\mathfrak{T}$-category $\mathbf{C}$ is a $\mathcal{C}$-category if all $\mathbf{C}\left(c, c^{\prime}\right)$ are in $\mathcal{C}$. For any such $\mathbf{C}$ a free prolongation rel $\mathbf{C}_{0}$ of an $X: \mathbf{C} \rightarrow \mathcal{C}$ along an $A: \mathbf{C}_{0} \rightarrow \mathcal{C}$ is again in $\bigodot^{\mathbf{c}}$; the same is evidently true of free prolongations of contractions.

If by $\mathbf{C}_{0}$-cofibration in $\mathrm{e}^{\mathbf{c}}$ we mean a right infinite composition of free prolongations rel $\mathbf{C}_{0}$ we may thus draw the following conclusion.

Proposition 7.3. Any $X \rightarrow Y$ in $\mathrm{C}^{\mathbf{C}}$ may be factored as $X \rightarrow \tilde{Y} \rightarrow Y$ with $X \rightarrow \tilde{Y} a$ $\mathbf{C}_{0}$-cofibration in $\mathrm{e}^{\mathbf{C}}$ and $\tilde{Y} \rightarrow Y$ a homotopy equivalence rel $\mathbf{C}_{0}$.

COROLlary 7.4. If $\mathbf{C}, \mathbf{D}$ are -categories and $F: \mathbf{C} \rightarrow \mathbf{D}$ then, up to isomorphism, $\mathrm{Ho}_{0} \operatorname{lan}_{F}$ takes $\mathrm{Ho}_{0} e^{\mathbf{C}}$ into $\mathrm{Ho}_{0} e^{\mathbf{D}}$.

If $X$ is a space in $\mathscr{T}$ we write $S X$ for the geometrical realization of the singular complex of $X$. Then $S$ is a functor $T \rightarrow \mathcal{C} \mathcal{J}$ and is provided with a natural transformation $s_{X}: S X \rightarrow X$ which is, for all $X$, a weak homotopy equivalence. Moreover $S$ preserves finite products. Thus if $\mathbf{C}$ is a $\sigma_{\text {-category we may define a }}$ C-category $\tilde{\boldsymbol{S}} \mathbf{C}$ by $(\tilde{\boldsymbol{S}} \mathbf{C})_{0}=\mathbf{C}_{0} ;(\boldsymbol{S C})\left(c, c^{\prime}\right)=\boldsymbol{S}\left(\mathbf{C}\left(c, c^{\prime}\right)\right)$, composition being given by

$$
\tilde{S} \mathbf{C}\left(c, c^{\prime \prime}\right) \times \tilde{S} \mathbf{C}\left(c, c^{\prime}\right) \approx S\left(\mathbf{C}\left(c^{\prime} \cdot c^{\prime \prime}\right) \times \mathbf{C}\left(c, c^{\prime}\right)\right) \rightarrow S\left(\mathbf{C}\left(c, c^{\prime \prime}\right)\right) .
$$

From $s$ we may construct a weak $H$-equivalence $\tilde{s}_{\mathbf{C}}: \tilde{S} \mathbf{C} \rightarrow \mathbf{C}$. 
If $X: \mathbf{C} \rightarrow \mathcal{T}$ define $\hat{S} X: \hat{S} \mathbf{C} \rightarrow \mathcal{T}$ by $(\hat{S} X)_{c}=S X_{c}$, with $\tilde{S} \mathbf{C}\left(c, c^{\prime}\right) \rightarrow X_{c^{\prime}}^{X_{c}}$ determined by adjunction from the commutative diagram

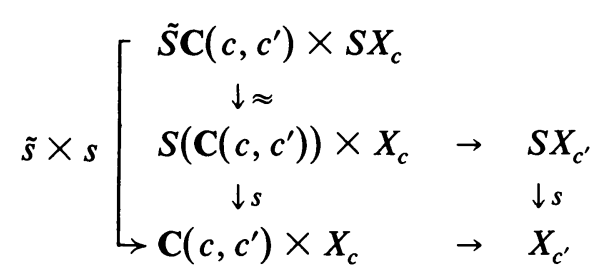

which also constructs for us a morphism $\hat{s}_{X}: \hat{S} X \rightarrow \tilde{s}^{*} X$, natural in $X$, which is for each $X$ a weak homotopy equivalence in $\mathcal{T}^{\tilde{s}}$.

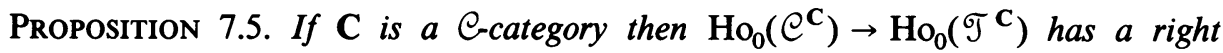
adjoint.

This is of course given by the composition

$$
\mathrm{Ho}_{0}\left(\mathcal{T}^{\mathbf{C}}\right) \rightarrow \mathrm{Ho}_{0}\left(e^{\tilde{s} \mathbf{C}}\right) \rightarrow \mathrm{Ho}_{0}\left(e^{\mathbf{c}}\right),
$$

the second functor being the inverse whose existence is guaranteed by Corollary 7.4.

This right adjoint makes $\mathrm{Ho}_{0}\left(e^{\mathbf{c}}\right)$ a weak category of fractions of $\mathrm{Ho}_{0}\left(\sigma^{\mathbf{C}}\right)$ with respect to weak homotopy equivalences and, proceeding as in Theorem 4.8, leads to the following theorem.

THEOREM 7.6. (i) For any $\mathbf{C}, \mathrm{Ho}_{0} \sigma \mathbf{C}$ admits a calculus of left fractions $\mathrm{Ho}_{w} \sigma \mathbf{C}_{\text {with }}$ respect to its weak homotopy equivalences.

(ii) If $F: \mathbf{C} \rightarrow \mathbf{D}$ then $F^{*}$ defines a functor $\mathrm{Ho}_{w} F^{*}: \mathrm{Ho}_{w} \mathcal{T}^{\mathbf{D}} \rightarrow \mathrm{Ho}_{w} \mathcal{J}^{\mathbf{C}}$ with adjoints

$$
\mathrm{Ho}_{w} \operatorname{lan}_{F}+\mathrm{Ho}_{w} F^{*}+\mathrm{Ho}_{w} \operatorname{ran}_{F}
$$

where $\mathrm{Ho}_{w} \mathcal{T}^{\mathbf{C}}$ is the category of fractions whose existence is guaranteed by (i).

(iii) If $F$ is a weak $H$-equivalence then $\mathrm{Ho}_{w} F^{*}$ is an equivalence of categories.

(iv) If $\mathbf{C}$ is a -category, $X \in \mathcal{C}^{\mathbf{C}}, Y \in \mathcal{T}^{\mathbf{c}}$, then $\mathrm{Ho}_{w} \mathcal{\sigma}^{\mathbf{c}}(X, Y) \approx \mathrm{Ho}_{0} \mathcal{T}^{\mathbf{C}}(X, Y)$. In particular, $\mathrm{Ho}_{0} \mathrm{e}^{\mathbf{C}}=\mathrm{Ho}_{w} \mathrm{e}^{\mathbf{C}} \rightarrow \mathrm{Ho}_{w} \mathcal{T}^{\mathbf{C}}$ is an equivalence of categories.

8. Basepoints. Starting with the category $\mathcal{T}$. of pointed spaces, i.e., the commacategory $(* \downarrow \mathcal{T})$, we may develop a functor calculus and homotopy theory in analogy with those just presented for $\mathcal{T}$. It is not necessary to do this in extenso; rather it will suffice to indicate the several differences between these theories.

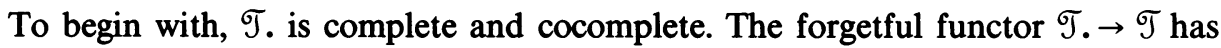
the left adjoint $X \mapsto X^{+}=(* \rightarrow X \sqcup *)$ and preserves pushouts and directed colimits; coproducts in $\mathcal{T}$. are of course given by " $\vee$ ". Moreover $\mathcal{T}$. is monoidal closed with respect to the smash product " $\wedge$ "; we denote the corresponding function spaces by $Y^{\cdot X}$.

Continuous functors $\mathbf{C} \rightarrow \mathcal{T}$. defined in the obvious way are the objects of the category $\mathcal{T}^{\mathrm{C}}{ }^{\mathrm{C}}$. For suitable $X, Y$ we define (cf. (1.2))

$$
\left(X \dot{\otimes}_{\mathbf{C}} Y\right)_{b d}=\int^{c} X_{b c} \wedge Y_{c d}, \quad \operatorname{Hom}_{\mathbf{C}}(X, Y)_{b d}=\int_{c} Y_{c d}^{\cdot X_{b c}} .
$$


If $F: \mathbf{C} \rightarrow \mathbf{D}$ we have, replacing (1.6),

$$
\begin{gathered}
F^{*} Y \approx \mathbf{D}^{+} \circ(1 \times F) \otimes_{\mathbf{D}} Y \approx \operatorname{Hom}_{\mathbf{D}}\left(\mathbf{D}^{+} \circ(F \times 1), Y\right), \\
\operatorname{Lan}_{F} X=\mathbf{D}^{+} \circ(F \times 1) \otimes_{\mathbf{C}} X, \quad \operatorname{Ran}_{F} X=\operatorname{Hom}_{\mathbf{C}}\left(\mathbf{D}^{+} \circ(1 \times F), X\right) .
\end{gathered}
$$

Homotopies in $\mathcal{T}^{\mathbf{C}}$ are morphisms $X \wedge I^{+} \rightarrow Y$ or $X \rightarrow Y^{\cdot I^{+}}$. Homotopy equivalences, fibrations and cofibrations are defined accordingly and the remaining arguments and conclusions of $\$ \S 1-6$, modified to fit the pointed case, may be left for the reader to supply.

This does not quite exhaust the subject of basepoints; we are bound to consider their "quality" as well. Let us observe that $\mathcal{T}^{\mathbf{C}}=(* \downarrow \mathcal{T})^{\mathbf{C}} \approx\left(* \downarrow \mathcal{T}^{\mathbf{C}}\right)$. Thus for any closed $\mathbf{C}^{\prime} \subset \mathbf{C}$ there is a full subcategory of $\mathcal{T}^{\mathbf{C}}$ containing those functors for which the basepoint $* \rightarrow X$ is a cofibration rel $\mathbf{C}^{\prime}$ in $\mathcal{T}^{\mathbf{C}}$. The standard argument (i.e., in the case $\mathbf{C}=\mathbf{1}$ ) proves also the following assertion.

Proposition 8.1. Suppose that $X, Y$ in $\mathcal{T}^{\mathbf{C}}$ have basepoints cofibered rel $\mathbf{C}^{\prime}$ and that $f: X \rightarrow Y$ in $\mathcal{T}^{\mathbf{C}}$ is a homotopy equivalence rel $\mathbf{C}^{\prime}$ in $\mathcal{T}^{\mathbf{C}}$. Then $f$ is also a homotopy equivalence rel $\mathbf{C}^{\prime}$ in $\mathcal{T}^{\mathbf{C}}$.

In the absolute case $\mathbf{C}^{\prime}=\mathbf{C}$ we shall say that an $X$ in $\mathcal{T}^{\mathbf{C}}$ is well pointed if $* \rightarrow X$ is a cofibration in $\mathcal{T}^{\mathbf{C}}$ and shall write $\mathcal{T}_{\mathbf{C}}^{\mathbf{C}} \subset \mathcal{J}^{\mathbf{C}}$ for the full subcategory of well-pointed functors. For any $X$ in $\mathcal{T}^{\mathbf{C}}$ we may write $X^{\#}$ for the mapping cylinder in $\mathcal{T}^{\mathbf{C}}$ of $* \rightarrow X$. This mapping cylinder lies in $\mathcal{T}_{\mathbf{m}}^{\mathbf{C}}$. For any $\mathbf{C}^{\prime} \subset \mathbf{C}$ it follows from Proposition 8.1 that if $X$ has basepoint cofibered rel $\mathbf{C}^{\prime}$ then the canonical morphism $X^{\#} \rightarrow X$ is a homotopy equivalence rel $\mathbf{C}^{\prime}$ in $\mathcal{T}^{\mathbf{C}}$.

Proposition 8.2. $X \rightarrow X^{\#}$ gives a right adjoint to the functors $\mathrm{Ho}_{\mathbf{C}^{\prime}} T_{\square}^{\mathbf{C}} \rightarrow \mathrm{Ho}_{\mathbf{C}^{\prime}} \sigma . \mathbf{C}^{\mathrm{C}}$ induced by inclusion.

We might, at this point, have introduced the subcategories of functors with basepoints cofibered rel $\mathrm{C}^{\prime}$; Proposition 8.1 shows that the corresponding homotopy categories would be equivalent to $\mathrm{Ho}_{\mathbf{C}^{\prime}} \sigma_{\mathbf{m}}^{\mathbf{C}}$.

The categories $\mathcal{T}_{\mathbf{D}}^{\mathbf{C}}$ lack most of the completeness properties of $\mathcal{T}_{.} \mathbf{C}$. Nevertheless the results on the existence of adjoint functors in homotopy may be recovered because of $\$ 2$ and the following lemma.

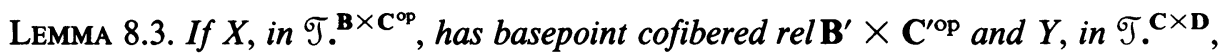
is $\mathbf{C}^{\prime} \times \mathbf{D}^{\prime}$-cofibrant then $X \dot{\otimes}_{\mathbf{C}} Y$ has basepoint cofibered rel $\mathbf{B}^{\prime} \times \mathbf{D}^{\prime}$.

This is an immediate consequence of Proposition 4.1 and the fact that

is a pushout in $\mathcal{T} \mathbf{B} \times \mathbf{D}$.

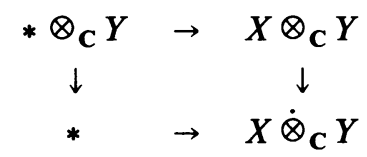

For brevity we confine ourselves to the statement of the existence of homotopy Kan extensions in the well-pointed case.

THEOREM 8.4. If $F:\left(\mathbf{C}, \mathbf{C}^{\prime}\right) \rightarrow\left(\mathbf{D}, \mathbf{D}^{\prime}\right)$ then $\mathrm{Ho}_{\mathbf{C}^{\prime}, \mathbf{D}^{\prime}} F^{*}$ takes $\mathrm{Ho}_{\mathbf{D}^{\prime}} \mathcal{T}^{\mathbf{D}}$ to $\mathrm{Ho}_{\mathbf{C}^{\prime}} \mathcal{T}_{\mathbf{q}}^{\mathbf{C}}$, with adjoints on both sides. 
By Lemma 8.3 the left adjoint is, up to isomorphism, just the restriction of $\mathrm{Ho}_{\mathbf{C}^{\prime}, \mathbf{D}^{\prime}} \operatorname{lan}_{F}$. The right adjoint is $\mathrm{Ho}_{\mathbf{C}^{\prime}, \mathbf{D}^{\prime}} \operatorname{ran}_{F}$ composed with the functor $X \mapsto X^{\#}$.

9. Half-exact functors. Let us denote by $\mathcal{T}_{0}$ the full subcategory of $\mathcal{T}$. containing the pathwise connected spaces. The full subcategory $\mathrm{Ho}_{w} \mathcal{T}_{0}^{\mathbf{C}} \subset \mathrm{Ho}_{w} \sigma^{\mathbf{C}} \mathbf{C}$ is closed under coproducts and weak pushouts. A functor $\left(\mathrm{Ho}_{w} \mathcal{T}_{0}^{\mathbf{C}}\right)^{\text {op }} \rightarrow$ Sets is half-exact if it takes coproducts in $\mathrm{Ho}_{w} \mathcal{T}_{0}^{\mathbf{C}}$ to products and weak pushouts to weak pullbacks.

THEOREM 9.1. A functor $\left(\mathrm{Ho}_{w} \sigma_{0}^{\mathbf{C}}\right)^{\mathrm{op}} \rightarrow$ Sets is representable if and only if it is half-exact.

The case $\mathbf{C}=\mathbf{1}$ is of course Brown's theorem. Representable functors are clearly half-exact. The converse depends on a theorem of category theory (cf. [7]).

CATEgorical Theorem. Suppose that $Q$ is a category supplied with coproducts and weak pushouts and possessing a set $\mathbf{S}$ of objects such that

(i) $\mathbf{S}$ is left adequate.

(ii) There is a cardinal number $\beta_{0}$ such that if $\beta$ is a regular cardinal greater than $\beta_{0}$ and $X: \beta \rightarrow \mathbb{Q}$ then $X$ has a weak colimit $\bar{X}$ such that, for any $S \in \mathbf{S}$, $\operatorname{colim}_{\alpha<\beta} \mathcal{Q}\left(S, x_{\alpha}\right) \rightarrow \mathcal{Q}(S, \bar{X})$ is bijective.

Then every half-exact functor $\mathbb{Q}^{\mathrm{op}} \rightarrow$ Sets is representable.

Thus to prove Theorem 9.1 we need only find a suitable set $\mathbf{S}$ in $\mathrm{Ho}_{w} \mathcal{T}_{0} \mathbf{C}$. By Theorem 7.6 we may without loss of generality suppose that $\mathbf{C}$ is a $\mathrm{C}$-category. We set $\mathbf{S}=\left\{\mathrm{Ho}_{0} \operatorname{lan}_{c} S^{n} ; c \in \mathbf{C}_{0}, n=1,2, \ldots\right\}$. Note that each of these is a functor

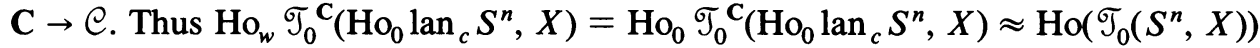
$=\pi_{n} X$ and it is clear that $\mathbf{S}$ is left adequate.

The boundedness condition, of course, follows immediately from Proposition 6.6.

\section{REFERENCES}

1. D. W. Anderson, Axiomatic homotopy theory (preprint).

2. G. Baumslag, E. Dyer and A. Heller, The topology of discrete groups, J. Pure Appl. Algebra 16 (1980), 1-47.

3. A. K. Bousfield and D. M. Kan, Homotopy limits, completions and localizations, Lecture Notes in Math., vol. 304, Springer-Verlag, Berlin, Heidelberg and New York, 1973.

4. A. Heller, Abstract homotopy in categories of fibrations and the spectral sequence of Eilenberg and Moore, Illinois J. Math. 16 (1972), 454-474.

5. __ Adjoint functor and bar constructions, Adv. in Math. 12 (1974), 8-31.

6. On the homotopy theory of topogenic groups and groupoids, Illinois J. Math. 24 (1980), $576-605$.

7. On the representability of homotopy functors, J. London Math. Soc. (2) 23 (1981), 551-562.

8. S. Mac Lane, Categories for the working mathematician, Graduate Texts in Math., Vol. 5, Springer-Verlag, New York, Heidelberg and Berlin, 1971.

9. D. MacDuff, On the classifying spaces of discrete monoids, Topology 18 (1979), 313-320.

10. D. Quillen, Homotopical algebra, Lecture Notes in Math., vol. 43, Springer-Verlag, Berlin, Heidelberg and New York, 1967.

11. G. Segal, Classifying spaces and spectral sequences, Inst. Hautes Etudés Sci. Publ. Math. 34 (1968), $105-112$.

12. N. Steenrod, Milgram's classifying space of a topological group, Topology 7 (1968), 135-152.

13. R. Vogt, Homotopy limits and colimits, Math. Z. 134 (1973), 11-52.

14. , Commuting homotopy limits, Math. Z. 153 (1977), 59-82.

Department of Mathematics, City University of New York Graduate Center, New York, NEW YORK 10036 